\title{
A note on combining correlations
}

\author{
RICHARD A. CHARTER \\ Department of Veterans Affairs Medical Center, Long Beach, California \\ and \\ RALPH A. ALEXANDER \\ The University of Akron, Akron, Ohio
}

\begin{abstract}
Equations for combining correlation coefficients (Pearson's $r$ ) on two or more samples are provided. These equations generally produce different values than those produced using averaging methods. The proper situations in which to use the combining or averaging methods are discussed. Data from the WAIS-R manual (Wechsler, 1981) is used as an example.
\end{abstract}

Researchers faced with attempting to estimate a correlation $(r)$ based on an aggregation of sample $r$ s usually either average the observed $r$ s (Guilford \& Fruchter, 1978) or use an average based on Fisher's $z$ transformation (Silver \& Dunlap, 1987). There are also other, less well known methods of finding an approximately unbiased estimator of the population correlation (Alexander, 1990). Averaging methods are appropriate when several different samples are independently drawn from the same population and the interest is in aggregating those values.

Another instance in which averaging is not the method of choice is when a number of samples have been drawn and each sample is presumed to be drawn from a different segment of the same population (by stratified random sampling) or when a single sample is drawn and then directly stratified. In this case, the interest is in computing the correlation in the unstratified sample when the only data available are means, standard deviations, sample sizes, and $r \mathrm{~s}$ from each of the stratification groups.

Sockloff (1975) derived equations to find the exact correlation coefficient for two samples combined on the basis of knowledge of the means, standard deviations, sample sizes, differences between subgroup means, and the pooled within-subgroup correlation coefficient (requiring knowledge of the within-subgroup sums of cross-products). The purpose of this note is to extend Sockloff's equations and present the equations for calculating the correlation for a combination of any number of sample $r$ s using only the subgroup means, standard deviations, sample sizes, and $r \mathrm{~s}$.

\section{EQUATIONS}

The raw score formula for calculating $r$ between variables $X$ and $Y$ is

$r=[N \Sigma X Y-(\Sigma X)(\Sigma Y)] /\left\{\left[\left(N \Sigma X^{2}-(\Sigma X)^{2}\right]\left[\left(N \Sigma Y^{2}-(\Sigma Y)^{2}\right]\right\}^{1 / 2}\right.\right.$.

Address all correspondence to R. A. Charter, Psychology Service (116B), Department of Veterans Affairs Medical Center, 5907 East Seventh Street, Long Beach, CA 90822, or to R. A. Alexander, Department of Psychology, University of Akron, Akron, OH 44325.
The following equations provide the sums to be placed in the above formula to find the combined $r$ :

$$
\begin{aligned}
N & =\Sigma n_{i} \\
\Sigma X & =\Sigma\left(n_{i} \overline{X_{i}}\right) \\
\Sigma Y & =\Sigma\left(n_{i} \overline{Y_{i}}\right) \\
\Sigma X^{2} & =\Sigma n_{i}\left(\overline{X_{i}^{2}}+S_{x_{i}}^{2}\right) \\
\Sigma Y^{2} & =\Sigma n_{i}\left(\overline{Y_{i}^{2}}+S_{y_{i}}^{2}\right) \\
\Sigma X Y & =\Sigma n_{i}\left(r_{i} S_{x_{i}} S_{y_{i}}+\overline{X_{i}} \overline{Y_{i}}\right),
\end{aligned}
$$

where $N$ is the total sample size, $n_{i}$ is the $i$ th subgroup sample size, $r_{i}$ is the $i$ th subgroup correlation between $X$ and $Y, \bar{X}_{i}$ and $\bar{Y}_{i}$ are the $i$ th sample means, and $S_{x_{i}}$ and $S_{y_{i}}$ are the $i$ th sample standard deviations.

These equations assume that the standard deviations were obtained with division by $n$, not by $n-1$. If the $S$ s were obtained by division by $n-1$, then multiplying each $S_{i}^{2}$ by $\left(n_{i}-1\right) / n_{i}$ before entering them in the above equations will adjust them appropriately.

\section{DISCUSSION}

The equations provided here give the exact value for the combination of subgroup $r$. Using these equations, one can calculate the $r$ that would have been obtained if the raw data had been analyzed as a single group.

The combining method will give results different from averaging methods to the extent that the stratification/grouping variable is itself correlated with either the $X$ or $Y$ variable, with the most extreme case being where the sample is stratified on one of the two variables.

As an example of the use of the combining method, the WAIS-R manual (Wechsler, 1981) gives nine correlation matrices (WAIS-R Table 15), each for a different age group. Wechsler included a table (WAIS-R Table 16) of the average $r$ s from the nine matrices. That table reports an average $r$ between the Verbal IQ (VIQ) and Performance IQ (PIQ) of .74. However, using the VIQ and PIQ summary statistics (WAIS-R Table 7), the combining equations result in a combined sample $r$ of .68 . An $r$ of .68 is what would have been obtained if all the original raw data for all nine age groups were correlated as a single group.

\section{REFERENCES}

Alexander, R. A. (1990). A note on averaging correlations. Bulletin of the Psychonomic Society, 28, 335-336. 
Guilford, J. P., \& Fruchter, B. (1978). Fundamental statistics in psychology and education (6th ed.). New York: McGraw-Hill.

Silver, N. C., \& DUNLAP, W. P. (1987). Averaging correlation coefficients: Should Fisher's $z$ transformation be used? Journal of Applied Psychology, 72, 146-148.

SockLOFF, A. L. (1975). Behavior of the product-moment correlation coefficient when two heterogeneous subgroups are pooled. Educational \& Psychological Measurement, 35, 267-276.

Wechsler, D. (1981). WAIS-R manual. San Antonio: Psychological Corporation.

(Manuscript received October 6, 1992.) 